\title{
迪美唑在光催化剂 $\mathrm{TiO}_{2}$ 表面吸附特征及降解机理 的理论研究
}

徐伯华 ${ }^{1}$, 张福兰 ${ }^{1}$, 魏维 ${ }^{2}$, 陈晓 ${ }^{2}$, 李来才 ${ }^{2 *}$, 田安民 ${ }^{3}$

1. 长江师范学院化学化工学院, 涪陵 408100

2. 四川师范大学化学与材料科学学院, 成都 610066

3. 四川大学化学学院, 成都 610064

*通讯作者, E-mail: lilcmail@163.com

收稿日期：2018-06-01; 接受日期：2018-08-03; 网络版发表日期：2018-11-20

四川省自然科学基金(编号: 2014JY0099)资助项目

摘要本文采用密度泛函理论研究了迪美唑在锐钛矿 $\mathrm{TiO}_{2}(101)$ 和 $(001)$ 晶面的吸附和降解反应机理, 分别研究 了真空和中性水溶剂条件下, 迪美唑在锐钛矿表面吸附的稳定性特征, 理论优化了最稳定的吸附构型. 研究发现 在两种条件下, 迪美唑均能吸附在 $\mathrm{TiO}_{2}$ 表面, 吸附过程产生的氢键能增强吸附结构的稳定性, 稳定的吸附构型使 得迪美唑的 $\mathrm{C}-\mathrm{N}$ 键变长, 有利于发生开环降解反应. 本文还研究了迪美唑在锐钛矿 $\mathrm{TiO}_{2}$ 两个晶面上的开环降解反 应机理. 研究发现, 在 $\mathrm{TiO}_{2}(101)$ 晶面迪美唑开环降解所需反应活化能较高, 很难发生, 而在(001)晶面迪美唑开环可 在热反应条件下进行. 在水溶剂条件下, 迪美号在锐钛矿 $\mathrm{TiO}_{2}$ 晶面上的降解反应活化能有所降低, 可见溶剂条件 能促进降解反应的进行.

关键词密度泛函理论, $\mathrm{TiO}_{2}$ 光催化剂, 迪美唑, 降解, 反应机理

\section{1 引言}

迪美唑(DMZ)是一类在禽畜养殖业中广泛使用的 硝基咪唑类抗生素 ${ }^{[1]}$, 主要用于干预和治疗受到细菌 感染和原生动物感染的家禽和猪 ${ }^{[2]}$. 由于迪美唑及其 代谢产物具有环状结构，有基因毒性、致癌和致突变 的副作用 ${ }^{[3]}$, 包括中国在内的许多国家已禁止其作为 饲料添加剂, 也不允许在食物中残留 ${ }^{[4]}$. 迪美唑作为一 种难降解的有机物，传统的生物降解方法对其几乎没 有降解效果 ${ }^{[5,6]}$, 导致其最终进入水体. 2013年, Leung
等 ${ }^{[7]}$ 对中国13个城市的自来水厂的133个样品进行了 药物浓度的检测和风险评估， $89 \%$ 的样品都检测到迪 美唑的存在, 浓度为6.9 17.7 ng/L, 对贞幼儿的健康造 成了极大的威胁. 目前处理迪美唑的方法主要为活性 炭吸附, 但这只是将其与环境分离, 并未进行降解 ${ }^{[8]}$.

$\mathrm{TiO}_{2}$ 光催化是一种极具潜力的氧化技术, 其反应 条件温和、操作简单、无二次污染, 在难降解有机物 处理方面具有极大的应用潜力 ${ }^{[9]}$. 一系列的研究表明, $\mathrm{TiO}_{2}$ 光催化技术对含氮杂环结构的抗生素有着良好的 处理效果, 研究表明 $\mathrm{TiO}_{2}$ 可有效降解莫西沙星 ${ }^{[10]}$. 高乃

引用格式: Xu B, Zhang F, Wei W, Chen X, Li L, Tian A. Adsorption characteristics and degradation mechanism of DMZ on photocatalyst $\mathrm{TiO}_{2}$ surface: a density functional theoretical study. Sci Sin Chim, 2019, 49: 368-379, doi: 10.1360/N032018-00127 
云等 ${ }^{[11]}$ 用 $\mathrm{TiO}_{2}$ 除去水中的磺胺甲恶唑，羟基自由基的 氧化作用在其中占主导地位. Lamine等 ${ }^{[12]}$ 以二氧化钠 为光催化剂, 研究了光催化降解甲硝唑的过程, 并考察 了酸碱度、光催化浓度等因素对光催化降解的影响. Sánchezd-Polo等 ${ }^{[13]}$ 用 $\gamma$ 射线对迪美唑进行降解, 效率约 为 $70 \%$. 陈冬梅等 ${ }^{[1]}$ 的研究表明, $\mathrm{TiO}_{2}$ 对迪美唑的降解 速率与溶剂环境和酸碱度有关. 抗生素的光催化降解 机理十分复杂，目前尚不明确硝基咪唑类抗生素的具 体降解过程. 常睿等 ${ }^{[14]}$ 结合光催化法与生物法来降解 咪唑类离子液体, 并用高效液相色谱-质谱(HPLC-MS) 对氧化后的碎片进行分析, 发现含 $\mathrm{N}$ 杂环化合物的降 解起始于邻近 $\mathrm{N}$ 原子的碳氧化过程. 随着 $\mathrm{N}$ 原子上侧链 长度的增加, 由于空间位阻的影响, 使得咪唑环更难被 进攻 ${ }^{[15]}$. 鸟嘌呤也含有咪唑环结构, 侯若冰等 ${ }^{[16]}$ 研究 了8-看基鸟嘌呤开环反应的机理，表明开环反应中包 含咪唑环上 $\mathrm{N}-\mathrm{C}$ 键断裂和质子转移两个步骤. 本文以 锐钛矿 $\mathrm{TiO}_{2}$ 为光催化剂, 研究了真空和水溶液环境下, 迪美唑在锐钛矿 $\mathrm{TiO}_{2}$ (101)和(001)晶面上的吸附情况 和开环反应机理, 并以水为溶剂, 研究了溶剂化效应对 反应的影响, 希望能为相关抗生素的降解研究提供理 论信息.

\section{2 计算方法}

锐钛矿 $\mathrm{TiO}_{2}(101)$ 面的表面有两种 $\mathrm{Ti}$ 原子, 分别是 五配位的 Ti(5)和六配位的 Ti(6). 其表面有两种 $\mathrm{O}$ 原子, 分别是二配位的 $\mathrm{O}(2)$ 和三配位的 $\mathrm{O}(3)$, 如图 $1(\mathrm{a}, \mathrm{b})$ 所 示. 基于板厚对表面能影响的初步研究，(101)晶面采 用3层的模型可以在计算时间和精度之间取得平衡 ${ }^{[17]}$. 以 $X Y$ 平面设置周期性边界条件, $Z$ 方向加 $15 \AA$ 的真空 层. 超晶胞由 $1 \times 3 \times 3$ 个单元的 108 个原子组成, 大小为 $10.89 \AA \times 11.33 \AA \times 25.00 \AA$. 在中性溶液环境中, 用Universal力场, 按照 $1 \mathrm{~g} / \mathrm{cm}^{3}$ 的密度约添加了 48 个 $\mathrm{H}_{2} \mathrm{O}$ 分子. 锐钛矿 $\mathrm{TiO}_{2}(001)$ 面的表面有五配位的 Ti(5), 六配位的 $\mathrm{Ti}(6)$ 位于内层, 表面有二配位的 $\mathrm{O}(2)$ 和三配位的 $\mathrm{O}(3)$, 如图1(c, d)所示. (001) 晶面采用一层的模型 ${ }^{[18]}$, 在 $X Y$ 平面设置周期性边界条件, $Z$ 方向加 $15 \AA$ 的真空层. 超 晶胞由 $3 \times 3 \times 1$ 个单元的 108 个原子组成, 大小为 $11.33 \AA \times 11.33 \AA \times 27.65 \AA$. 在中性溶液中, 用Universal 力场, 按照 $1 \mathrm{~g} / \mathrm{cm}^{3}$ 的密度约添加了 69 个 $\mathrm{H}_{2} \mathrm{O}$ 分子.

将迪美唑分子放于 $\mathrm{TiO}_{2}(101)$ 和(001)晶面之上，为
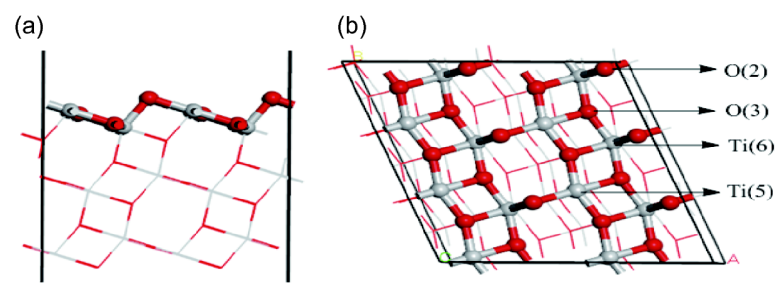

(c)

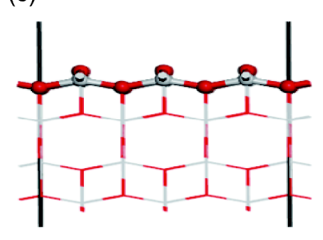

(d)

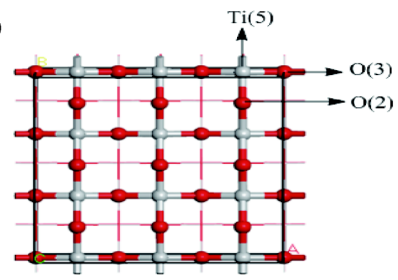

图 $1 \mathrm{TiO}_{2}(101)$ 和(001)晶面模型. (a, c) (101)和(001)晶面的 侧视图; (b, d) (101)和(001)晶面的 Z轴方向上的俯视图(网络 版彩图)

Figure 1 The models of $\mathrm{TiO}_{2}$ (101) and (001) surface. (a, c) Side views of the $\mathrm{TiO}_{2}$ (101) and (001) crystal faces, respectively; (b, d) Top views of the $\mathrm{TiO}_{2}(101)$ and (001) crystal faces in the $Z$-axis direction, respectively (color online).

了避免分子与表面间强烈的相互作用，二者之间的距 离设置为大于 $3.8 \AA$, 再将它们用ReaxFF力场进行分子 动力学计算 ${ }^{[19]}$. 分子动力学模拟用LAMMPS ${ }^{[20]}$ 程序在 NVE系综下进行, 弛豫步长为 $0.1 \mathrm{fs}$, 时长共 $10 \mathrm{ps}$. 在 LAMMPS弛豫结果基础上，选择局域极小点对应结构 进行密度泛函理论(DFT)的进一步结构优化.

本工作DFT所有结构优化都在自旋极化的平面波 基础上进行，电子交换相关能采用广义梯度近似 $(\mathrm{GGA})$ 的PBE ${ }^{[21]}$ 方法, K-point设置为 $1 \times 1 \times 1$, 平面波截 断能为 $380 \mathrm{eV}$, 能量的收玫标准为低于 $0.001 \mathrm{eV}$, 力的 收玫标准为低于 $0.05 \mathrm{eV} / \AA \AA$, 色散力在非共价结合的系 统中发挥了至关重要的作用, 而传统的DFT理论不足 以描述色散相互作用，近年来，一些修正方案的发展 用以弥补不足, 许多研究 ${ }^{[22-24]}$ 证明DFT-D3方法在非共 价相互作用的系统中表现出良好的计算效果, 因此本 文也采用了DFT-D3色散矫正计算. DFT计算会低估能 带值 ${ }^{[25]}$, 一些矫正方法可以得到良好的结果, 如DFT $+\mathrm{U}^{[26]}$, 本研究对 $(101)$ 表面采用 $U=7.0 \mathrm{eV}^{[27]}$, 对(001)表 面采用 $U=8.5 \mathrm{eV}^{[28]}$. 吸附能定义为吸附前后物质能量 的变化.

$$
E_{\text {ads }}=\left(E+E_{\text {sur }}\right)-E_{\text {adsorption }},
$$

其中 $E_{\text {adsorption }}$ 为吸附后体系的总能量, $E$ 为被吸附物质 能量, $E_{\text {sur }}$ 为吸附面的能量. 本文研究采用Material Stu- 
dio软件中的Dmol 模块进行，采用广义梯度近似的 GGA-PBE ${ }^{[21]}$ 描述相关函数; 核电子使用Dolg等 ${ }^{[29]}$ 和 Bergner等 ${ }^{[30]}$ 发明的ECP进行描述; 用可极化的 $\mathrm{DNP}^{[31]}$ 基组对原子轨道基组进行描述，截断半径大小定为 $3.7 \AA$, 自洽迭代能量收玫标准为 $1.0 \times 10^{-6} \mathrm{Ha}$, 力的误 差小于 $0.002 \mathrm{Ha} / \AA \AA$, 采用 $\mathrm{LST} / \mathrm{QST}^{[32]}$ 方法寻找过渡态, 通过频率分析来验证过渡态结构. 采用 $\mathrm{COSMO}^{[33,34]}$ 模 型, 选择水为溶剂来研究反应的溶剂化效应.

\section{3 计算结果}

\section{1 迪美唑在 $\mathrm{TiO}_{2}$ 表面的吸附}

我们优化了迪美唑分子结构以及 $\mathrm{TiO}_{2}(101)$ 和 (001)稳定晶面, 并基于LAMMPS分子动力学结构弛豫 和DFT进一步结构优化, 得到了迪美唑在 $\mathrm{TiO}_{2}(101)$ 和 (001)晶面上 5 种稳定吸附构型, 如图2和3所示. 此计算 得到的吸附能和吸附能隙列于表 1 中. 迪美唑在 $\mathrm{TiO}_{2}$ 的 吸附构型均为多位点吸附。咪唑环上的 $\mathrm{N}$ (3) 原子和 $\mathrm{C}(5)$ 原子上所连硝基氧原子能吸附在 $\mathrm{TiO}_{2}(101)$ 表面五 配位的 $\mathrm{Ti}(5)$ 原子上, $\mathrm{C}(2)$ 和 $\mathrm{N}$ (1)所连甲基上的 $\mathrm{H}$ 原子以 及 $\mathrm{C}(4)$ 上的 $\mathrm{H}$ 原子能与 $\mathrm{TiO}_{2}(101)$ 晶面二配位的 $\mathrm{O}(2)$ 原 子形成氢键, Zhang 等 ${ }^{[35]}$ 研究了染料 $/ \mathrm{TiO}_{2}$ 界面吸附, 发 现吸附过程中氢键作用增加了染料聚集物在 $\mathrm{TiO}_{2}$ 表面
的稳定性. Li等 ${ }^{[36]}$ 的研究表明, 胶原、聚乙烯吡咯烷酮 与 $\mathrm{TiO}_{2}$ 之间的氢键能增强纳米复合支架的稳定性. 由 此可见, 氢键的形成能够增加吸附构型的稳定性. 迪 美唑在 $\mathrm{TiO}_{2}(101)$ 晶面上的 5 种稳定吸附结构中, 并没 有发现其与三配位的 $\mathrm{O}(3)$ 原子形成氢键, 说明 $\mathrm{TiO}_{2}$ (101)晶面 $\mathrm{O}(2)$ 原子比 $\mathrm{O}(3)$ 原子活性更强. 此外由于迪 美唑分子与 $\mathrm{TiO}_{2}$ 表面的相互作用，使得 $\mathrm{TiO}_{2}$ 表面的某 些键长发生变化. $\mathrm{TiO}_{2}(001)$ 表面的吸附情况与(101)晶 面类似. 咪唑环上硝基的氧原子也能吸附在 $\mathrm{TiO}_{2}(001)$ 晶面五配位的 $\mathrm{Ti}(5)$ 原子上， $\mathrm{N}(1)$ 和 $\mathrm{C}(2)$ 所连甲基上的 $\mathrm{H}$ 原子, 以及 $\mathrm{C}(4)$ 上的 $\mathrm{H}$ 原子能与 $\mathrm{TiO}_{2}(001)$ 晶面二配位 的 $\mathrm{O}(2)$ 原子和三配位的 $\mathrm{O}(3)$ 原子形成氢键, 与 $\mathrm{TiO}_{2}$ (001) 晶面二配位的 $\mathrm{O}(2)$ 形成的氢键键长更短, 可能会 增强迪美唑分子与 $\mathrm{TiO}_{2}(001)$ 晶面的相互作用.

由表1可知, $\mathrm{A} 3$ 吸附方式的吸附能为 $1.67 \mathrm{eV}$, 吸附 能最大, 为最佳吸附构型, 在A3构型中, 迪美唑的 $\mathrm{N}(3)$ 原子吸附在 $\mathrm{TiO}_{2}(101)$ 晶面的 $\mathrm{Ti}(5)$ 位, 吸附距离为 $2.279 \AA$ ，迪美唑甲基上的两个 $\mathrm{H}$ 原子，以及迪美唑上 $\mathrm{C}(4)$ 连接的 $\mathrm{H}$ 原子均与 $\mathrm{TiO}_{2}(101)$ 晶面的 $\mathrm{O}(2)$ 原子形成 氢键, 键长分别为 $2.389 、 2.213$ 和 $1.916 \AA$. 由于 $\mathrm{TiO}_{2}$ (101)晶面的吸引, 咪唑环上 $\mathrm{N}(1)-\mathrm{C}(2)$ 键长由 $1.343 \AA$ 增长到 $1.358 \AA$. 含氮杂环化合物的降解一般起始于邻 近氮原子的碳氧化过程，迪美唑的 $\mathrm{N}(1)-\mathrm{C}(2)$ 键变弱有
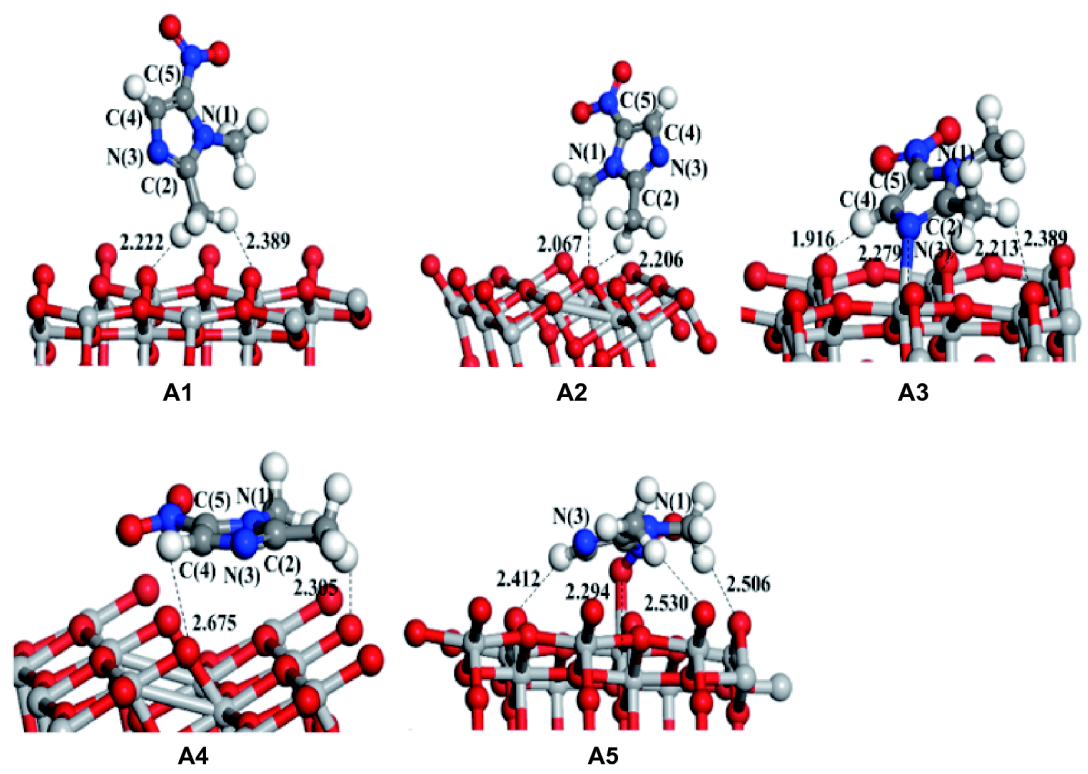

图 2 迪美唑在 $\mathrm{TiO}_{2}(101)$ 晶面上的吸附构型 $(\AA)$ (网络版彩图)

Figure 2 Adsorption configurations of $\mathrm{DMZ}$ on $\mathrm{TiO}_{2}(101)$ surface in vacuum ( $\AA$ ) (color online). 


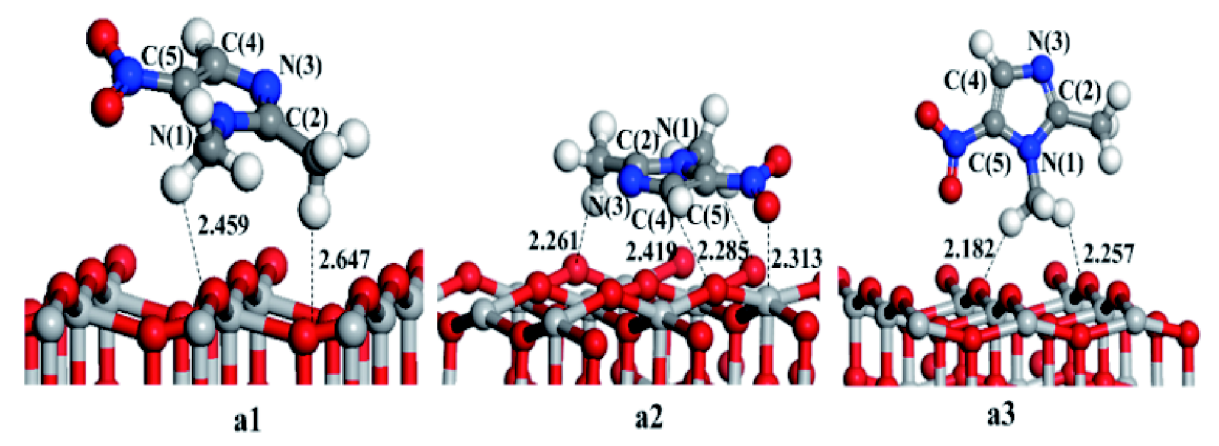

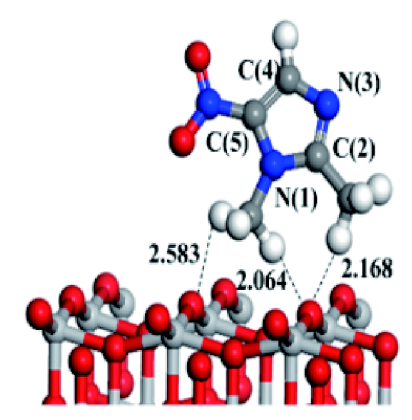

a4

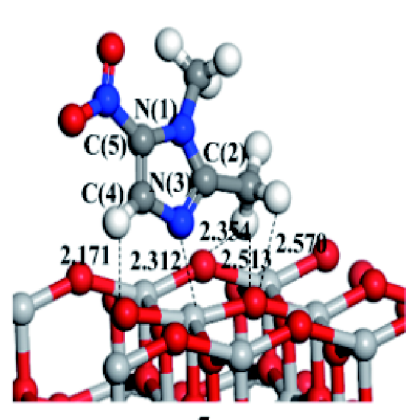

a5

图 3 迪美唑在 $\mathrm{TiO}_{2}(001)$ 晶面上的吸附构型 $(\AA)$ (网络版彩图)

Figure 3 Adsorption configurations of $\mathrm{DMZ}$ on $\mathrm{TiO}_{2}(001)$ surface in vacuum ( $\AA$ ) (color online).

表 1 迪美唑在 $\mathrm{TiO}_{2}$ 晶面吸附能 $(\mathrm{eV})$

Table 1 Adsorption energies of $\mathrm{DMZ}$ adsorbed on $\mathrm{TiO}_{2}$ surface

\begin{tabular}{|c|c|c|c|c|}
\hline \multirow{2}{*}{ 条件 } & \multicolumn{2}{|c|}{$\mathrm{TiO}_{2}(101)$ 晶面 } & \multicolumn{2}{|c|}{$\mathrm{TiO}_{2}(001)$ 晶面 } \\
\hline & 复合物 & $E_{\text {ads }}$ & 复合物 & $E_{\mathrm{ads}}$ \\
\hline \multirow{5}{*}{ 真空 } & $\mathrm{A} 1$ & 0.42 & a1 & 0.60 \\
\hline & $\mathrm{A} 2$ & 0.50 & $\mathrm{a} 2$ & 1.35 \\
\hline & $\mathrm{A} 3$ & 1.67 & a3 & 0.41 \\
\hline & A4 & 0.93 & $\mathrm{a} 4$ & 0.44 \\
\hline & A5 & 1.40 & a5 & 1.36 \\
\hline \multirow{5}{*}{ 水溶剂 } & B1 & 1.72 & b1 & 1.85 \\
\hline & B2 & 2.10 & b2 & 0.44 \\
\hline & B3 & 2.28 & b3 & 0.20 \\
\hline & B4 & 1.68 & b4 & 1.59 \\
\hline & B5 & 1.98 & b5 & 2.17 \\
\hline
\end{tabular}

利于羟基自由基的进攻. 迪美唑在 $\mathrm{TiO}_{2}(101)$ 晶面吸附 的次稳定构型为 $\mathrm{A} 5$, 吸附能为 $1.40 \mathrm{eV}$, 在 $\mathrm{A} 5$ 构型中, 迪美唑硝基上的O原子吸附在表面 $\mathrm{Ti}(5)$ 原子上，吸附 距离为 $2.294 \AA$, 迪美唑 $\mathrm{C}(2)$ 上所连甲基的 $\mathrm{H}$ 原子, 以及
$\mathrm{C}(4)$ 上的 $\mathrm{H}$ 原子均与 $\mathrm{TiO}_{2}(101)$ 晶面上 $\mathrm{O}(2)$ 原子形成较 弱的氢键, 键长分别为 $2.530 、 2.506$ 和 $2.412 \AA$. 在对咪 唑环的键长进行分析时发现 C(2)-N(1)和 C(4)-C(5)两 个双键的键长均有增长, $\mathrm{C}(2)-\mathrm{N}(1)$ 键长变长的原因可 能是由于 $\mathrm{C}(2)$ 所连甲基上的 $\mathrm{H}$ 原子与 $\mathrm{O}(2)$ 形成氢键的 缘故, 而 $\mathrm{C}(4)-\mathrm{C}(5)$ 键长变长则可能是由于迪美唑上的 硝基 $\mathrm{O}$ 原子和迪美唑 $\mathrm{C}(4)$ 上 $\mathrm{H}$ 原子与 $\mathrm{TiO}_{2}$ 晶面的相互 作用造成的. 总地来讲, 迪美唑上 $\mathrm{H}$ 原子易于与 $\mathrm{TiO}_{2}$ 表 面的O原子形成氢键, 使迪美唑分子吸附到 $\mathrm{TiO}_{2}$ 表面, 迪美唑 $\mathrm{N}(1)$ 上连的是甲基, 支链短、位阻小, 使得分 子上更多的原子能吸附到 $\mathrm{TiO}_{2}$ 表面.

迪美唑在 $\mathrm{TiO}_{2}(001)$ 晶面上的5种稳定吸附结构中, 由表 1 可知, $\mathrm{a} 5$ 吸附方式的吸附能为 $1.36 \mathrm{eV}$, 吸附能最 大, 为最佳吸附构型, 在 $\mathrm{a} 5$ 构型中, 迪美唑 $\mathrm{N}(3)$ 吸附在 $\mathrm{TiO}_{2}(001)$ 晶面 $\mathrm{Ti}(5)$ 原子上, 吸附距离为 $2.312 \AA$, 与迪 美唑吸附前的结构相比, 在构型a 5 中, 由于迪美唑 $\mathrm{N}(1)$ 原子与 $\mathrm{Ti}(5)$ 原子之间相互作用, 使得迪美唑的 N(1)$\mathrm{C}(2)$ 和 $\mathrm{N}(3)-\mathrm{C}(4)$ 键增长, 分别由原来的 1.343 和 $1.356 \AA$ 增长到 1.358 和 $1.364 \AA$, 迪美唑的 $\mathrm{N}(1)-\mathrm{C}(2)$ 键 变弱有利于羟基自由基的进攻, 对开环降解有利. 迪 
美唑在 $\mathrm{TiO}_{2}(001)$ 表面吸附的次稳定构型为 $\mathrm{a} 2$ ，吸附能 为 $1.35 \mathrm{eV}$ ，在构型 $\mathrm{a} 2$, 迪美唑上的硝基 $\mathrm{O}$ 原子吸附在 $\mathrm{TiO}_{2}(001)$ 表面 $\mathrm{Ti}(5)$ 原子上，吸附距离为 $2.313 \AA$ ，迪美 唑上 $\mathrm{C}(2)$ 所连甲基上的 $\mathrm{H}$ 原子，以及 $\mathrm{C}(4)$ 上的 $\mathrm{H}$ 原子均 与 $\mathrm{TiO}_{2}(001)$ 表面 $\mathrm{O}(2)$ 原子形成氢键, 键长分别为 $2.285 、 2.261$ 和 $2.419 \AA$, 有利于增加吸附的稳定性. 与 $\mathrm{TiO}_{2}(101)$ 晶面吸附结果相似, 在 $\mathrm{a} 2$ 构型中, 迪美唑的 $\mathrm{N}$ (1) $-\mathrm{C}(2)$ 键变弱, 有利于羟基自由基的进攻而开环 降解.

为了考察溶剂条件下的吸附特征，我们用相同的 方法优化了水溶液中迪美唑在 $\mathrm{TiO}_{2}$ 的(101)和(001)晶 面上的 5 种稳定的吸附构型, 如图4和5所示, 图中展示 了迪美唑的各个吸附构型和吸附距离. 在水溶液中, 由 于水分子的影响，与气相条件下相比，迪美唑分子与 $\mathrm{TiO}_{2}$ 表面原子的相互作用较弱, 吸附距离较远. 溶液 中的水分子上的 $\mathrm{O}$ 原子很容易吸附在 $\mathrm{TiO}_{2}$ 表面的 $\mathrm{Ti}(5)$ 原子上，水分子上的 $\mathrm{H}$ 原子和 $\mathrm{O}(2)$ 原子会形成氢键，因 $\mathrm{O}(3)$ 原子位置靠下，没有发现水分子中的 $\mathrm{H}$ 原子吸附 在 $\mathrm{TiO}_{2}$ 表面 $\mathrm{O}(3)$ 原子上. 此外, 迪美唑分子上的 $\mathrm{H}$ 原 子、硝基 $\mathrm{O}$ 原子和咪唑环上裸露的 $\mathrm{N}$ (3) 原子均会与水 分子形成氢键. 在水溶液中, 迪美唑在 $\mathrm{TiO}_{2}$ 的(101)和
(001)晶面上的吸附特征相似.

由表1可知, B3吸附结构的吸附能为 $2.28 \mathrm{eV}$, 吸附 能最大, 为最佳吸附构型. 与气相 $\mathrm{A} 3$ 吸附结构具有相 同的吸附特征. b5 吸附结构是迪美唑在 $\mathrm{TiO}_{2}(001)$ 表面 最稳定的吸附位，与气相条件下最稳定吸附B5 情况相 似，我们对比分析了气相和溶剂化条件下迪美唑在 $\mathrm{TiO}_{2}(001)$ 和(101)晶面的吸附特征, 发现最稳定吸附结 构特征是相似的. 在气相条件下迪美唑在 $\mathrm{TiO}_{2}(101)$ 和 (001)晶面上最稳定吸附构型分别为 $\mathrm{A} 3$ 和 $\mathrm{a} 5$, 在中性水 溶剂中, 迪美唑在 $\mathrm{TiO}_{2}(101)$ 和(001)晶面上最稳定吸附 构型分别为B3 和 b5. 迪美唑的 $\mathrm{N}(1)-\mathrm{C}(2)$ 键长相对于气 相条件下变得更长. 在水溶剂吸附模型中, 我们研究发 现水分子分布在迪美唑周围1.5 2.5 §回，说明水分 子与迪美唑分子间有较强的相互作用.

我们分别计算了气相和水溶剂条件下, $\mathrm{TiO}_{2}$ 的 (101)和(001)晶面的能带, 吸附后, $\mathrm{TiO}_{2}$ 价带能级和导 带能级均有所下降, 能隙变窄, 我们计算的 $\mathrm{TiO}_{2}$ 吸附前 的能隙结果为 $3.20 \mathrm{eV}$, 与实验值 $3.20 \mathrm{eV}^{[37]}$ 相吻合, 吸 附后, $\mathrm{A} 1 \sim \mathrm{A} 5$ 中的能隙值分别降到2.30、2.18、1.57、 $1.92 、 1.68 \mathrm{eV} ; \mathrm{B} 1 \sim \mathrm{B} 5$ 能隙分别降低至2.27、2.20、 2.06、2.39、2.30 eV; a1 a5 的能隙值分别降到 2.65 、

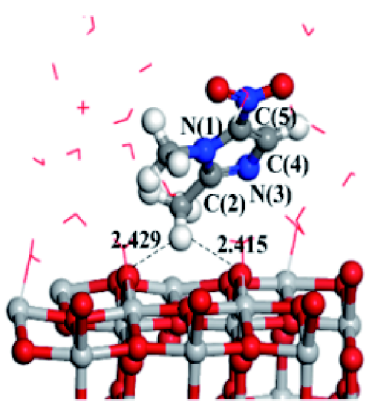

B1

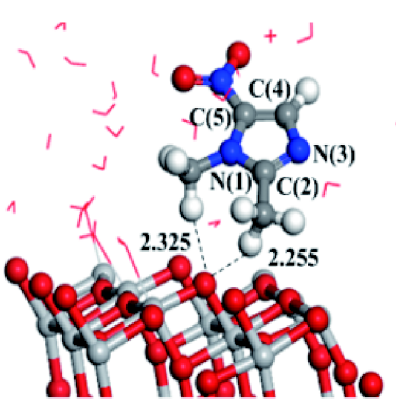

B2

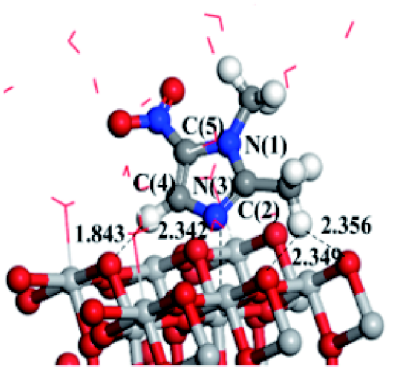

B3

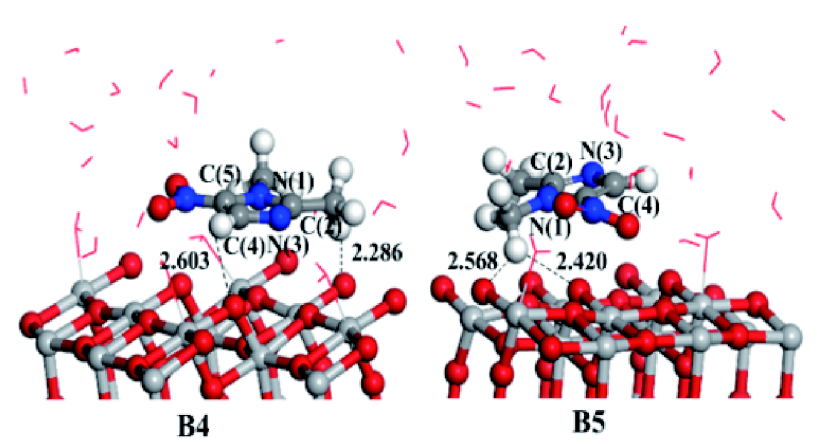

图 4 水溶液中迪美唑在 $\mathrm{TiO}_{2}(101)$ 晶面上的吸附构型 $(\AA)$ (网络版彩图)

Figure 4 Adsorption configurations of $\mathrm{DMZ}$ on $\mathrm{TiO}_{2}$ (101) surface in water solution ( $\AA$ ) (color online). 


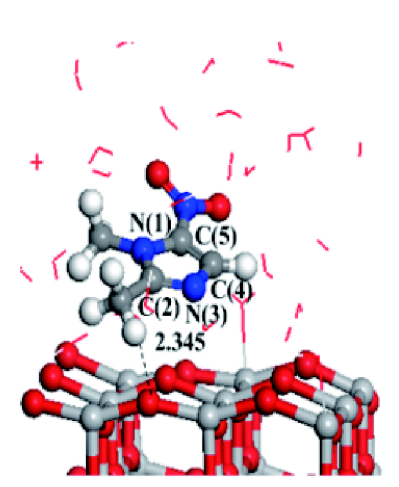

b1

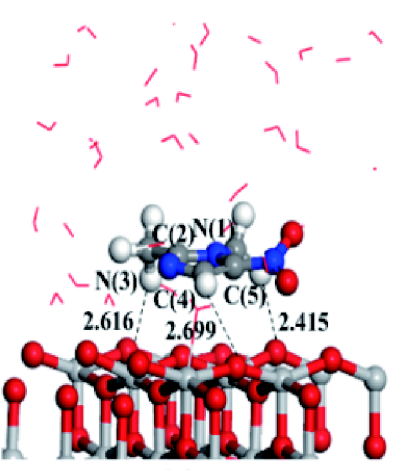

b2

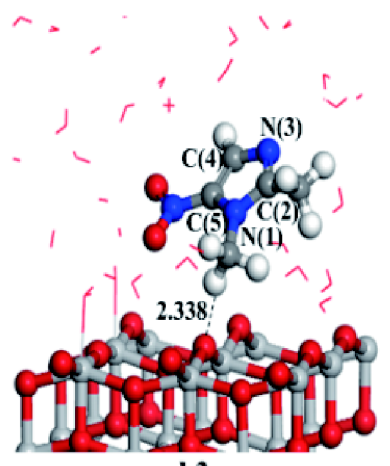

b3

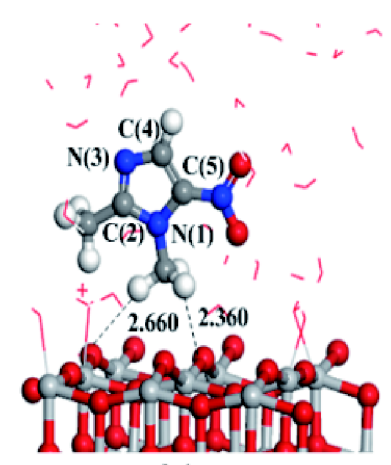

b4

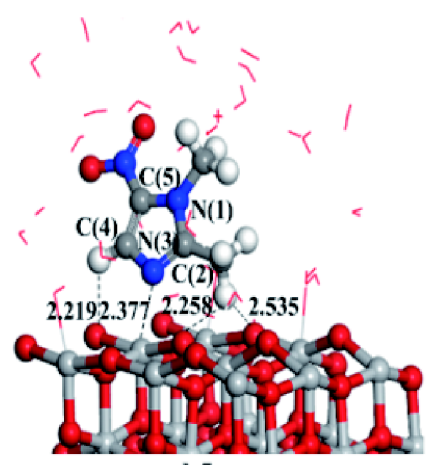

b5

图 5 水溶液中迪美唑在 $\mathrm{TiO}_{2}(001)$ 晶面上的吸附构型 $(\AA)$ (网络版彩图)

Figure 5 Adsorption configurations of $\mathrm{DMZ}$ on $\mathrm{TiO}_{2}(001)$ surface in water solution ( $\AA$ ) (color online).

1.63、2.65、2.52、1.53 eV; b1 b5 能隙分别降低至 $1.77 、 1.13 、 0.88 、 1.10$ 和 $0.69 \mathrm{eV}$ ，迪美唑在 $\mathrm{TiO}_{2}$ 的 (101)晶面上的吸附结构，其价带和导带间电子跃迁波 长在可见光范围之内 (可见光光子的能量范围约为 $1.62 \sim 3.11 \mathrm{eV})$, 可以有效利用可见光驱动迪美唑在 $\mathrm{TiO}_{2}$ 表面的降解.

\section{2 迪美唑在 $\mathrm{TiO}_{2}$ 表面的催化开环反应机理}

当 $\mathrm{TiO}_{2}$ 受到大于其禁带宽度能量的光子照射后, 激发产生电子和空穴, 电子有强还原性, 空穴有强氧化 性，一些电子和空穴分离并迁移到光催化剂表面的不 同位置, 电子将吸附在 $\mathrm{TiO}_{2}$ 表面的 $\mathrm{O}_{2}$ 还原为 $\mathrm{O}_{2}^{-}$, 空穴 将吸附在催化剂上的 $\mathrm{H}_{2} \mathrm{O}$ 氧化为 $\mathrm{OH}$ 自由基, 抗生素分 子即可被活性物质 $\mathrm{O}_{2}{ }^{-}$和 $\mathrm{OH}$ 自由基降解 ${ }^{[38]}$. 咪唑环的 开环是研究迪美唑类抗生素降解的重要环节 ${ }^{[14]}$, 开环 反应中包含咪唑环上 $\mathrm{N}-\mathrm{C}$ 键断裂和质子转移两个步 骤 ${ }^{[16]}$. 为了探究迪美唑在 $\mathrm{TiO}_{2}(101)$ 和(001)晶面上的开 环反应机理, 我们设计了两条反应路径, 并研究了溶剂 化影响. 本文分别从上述研究迪美唑在 $\mathrm{TiO}_{2}(101)$ 和
(001)晶面上吸附的稳定结构出发, 选取最稳定的A3和 a5 作为开环反应的起始物, 起始物受到催化剂表面 $\mathrm{OH}$ 自由基进攻后, 转化成 $R e$ 和 $R^{*}$. 在形成中间体 $R e$ 和 $\mathrm{Re}^{*}$ 的过程中, $\mathrm{C}(2)-\mathrm{N}(1)$ 键增长, 所以我们认为迪美唑 开环降解位置在咪唑环上C(2)-N(1)的断裂. 在反应通 道I中, 首先是断裂 $\mathrm{C}(2)-\mathrm{N}(1)$ 键, 然后羟基上的 $\mathrm{H}(1)$ 原 子转移到咪唑环的 $\mathrm{N}(3)$ 原子上, 在迪美唑 $\mathrm{C}(2)$ 原子处 形成烯醇式结构, 此后羟基上的 $\mathrm{H}(1)$ 原子直接向 $\mathrm{N}(3)$ 原子转移时会经过一个四元环结构的过渡态, 最后形 成产物; 反应通道II首先是羟基上的 $\mathrm{H}(1)$ 原子经过一 个四元环过渡态转移到咪唑环的 N(3) 原子上, 然后 $\mathrm{C}(2)-\mathrm{N}(1)$ 键断裂形成产物, 我们优化所得的反应各驻 点构型参数列于表 2 中, 反应结构图见图 6 .

在 $\mathrm{TiO}_{2}\left(\begin{array}{ll}0 & 0\end{array}\right)$ 晶面上, 反应通道 $\mathrm{I}$ 的机理为: $\mathrm{Re} \rightarrow \mathrm{TS} 1 \rightarrow \mathrm{M} 1 \rightarrow \mathrm{TS} 2 \rightarrow \mathrm{P}$, 在 Re中, 咪唑环上的 $\mathrm{N}(3)$ 原 子与 $\mathrm{TiO}_{2}(101)$ 表面 $\mathrm{Ti}(5)$ 原子之间的距离为 $2.602 \AA$, $\mathrm{TiO}_{2}$ 表面上的羟基自由基进攻迪美唑上 $\mathrm{C}(2)$ 原子, $\mathrm{C}(2)-\mathrm{O}(1)$ 键长为 $1.408 \AA, \mathrm{C}(2)-\mathrm{N}(1)$ 键长由原来的 $1.370 \AA$ 增加到 $1.499 \AA, \mathrm{N}(3)-\mathrm{C}(2)$ 键长由原来的 
表 2 迪美唑在 $\mathrm{TiO}_{2}(101)$ 晶面上反应过程中各驻点的构型参数 $(\AA)$

Table 2 Configuration parameters of each standing point in the reaction of $\mathrm{DMZ}$ on $\mathrm{TiO}_{2}(101)$ surface $(\AA)$

\begin{tabular}{|c|c|c|c|c|c|c|}
\hline 路径 & 化合物 & $\mathrm{N}(1)-\mathrm{C}(2)$ & $\mathrm{C}(2)-\mathrm{N}(3)$ & $\mathrm{C}(2)-\mathrm{O}(1)$ & $\mathrm{O}(1)-\mathrm{H}(1)$ & $\mathrm{N}(3)-\mathrm{H}(1)$ \\
\hline \multirow{5}{*}{ I } & $\operatorname{Re}$ & 1.499 & 1.488 & 1.408 & 1.029 & - \\
\hline & TS1 & 2.255 & 1.397 & 1.278 & 1.158 & - \\
\hline & M1 & 3.048 & 1.399 & 1.239 & 1.511 & - \\
\hline & TS2 & 3.248 & 1.399 & 1.292 & 1.831 & 1.963 \\
\hline & $\mathrm{P}$ & 3.176 & 1.371 & 1.261 & - & 1.083 \\
\hline \multirow{3}{*}{ II } & $\mathrm{TS} 3$ & 1.486 & 1.453 & 1.481 & 1.418 & 1.678 \\
\hline & M2 & 1.535 & 1.494 & 1.337 & - & 1.076 \\
\hline & TS4 & 2.256 & 1.423 & 1.240 & - & 1.079 \\
\hline
\end{tabular}

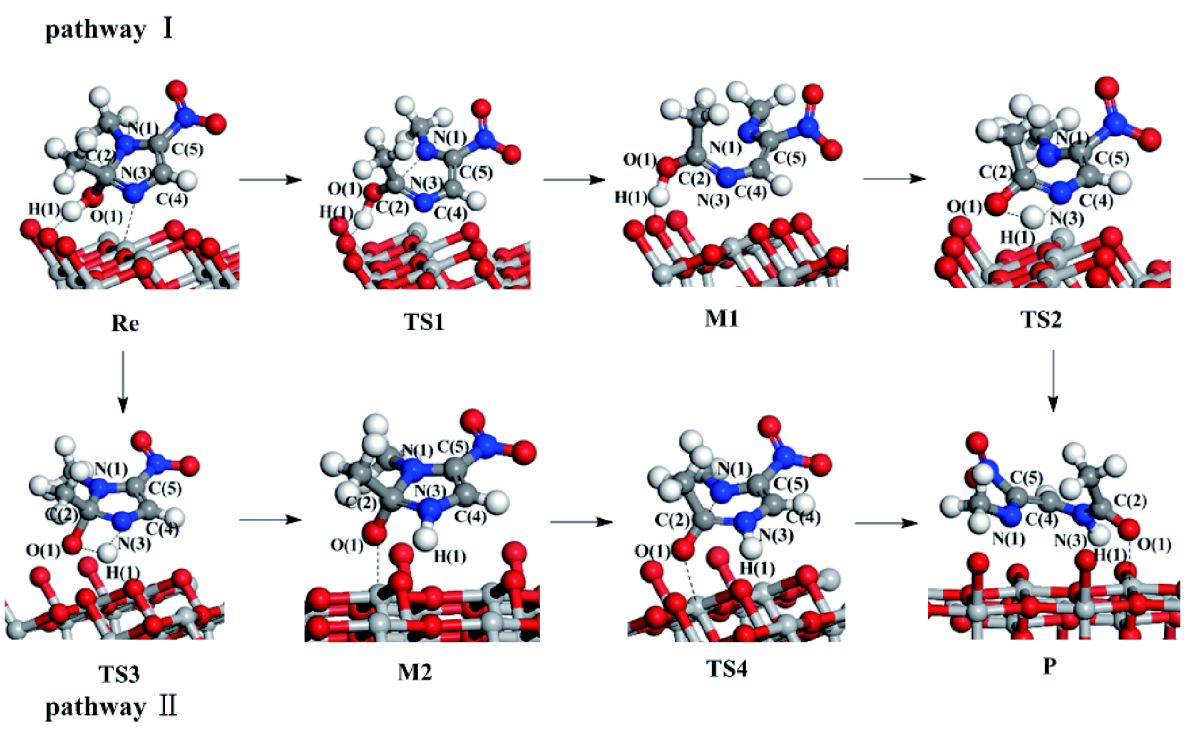

图 6 迪美唑在 $\mathrm{TiO}_{2}(101)$ 晶面上反应各驻点的构型(网络版彩图)

Figure 6 Configurations of each standing point in the reaction of $\mathrm{DMZ}$ on the $\mathrm{TiO}_{2}(101)$ surface (color online).

$1.343 \AA$ 拉长到 $1.488 \AA, \mathrm{C}(2)-\mathrm{N}(1)$ 键、 $\mathrm{N}(3)-\mathrm{C}(2)$ 键比 其咪唑环上的其他共价键都明显增长, $\mathrm{C}(2)-\mathrm{N}(1)$ 键又 比 $\mathrm{N}(3)-\mathrm{C}(2)$ 键稍长, 所以 $\mathrm{C}(2)-\mathrm{N}(1)$ 键断裂开环的可能 性最大. 在过渡态TS1中, N(1)-C(2)键开始断裂，键长 拉长到 $2.225 \AA$, 该步骤 $\mathrm{Re} \rightarrow \mathrm{TS} 1$ 的活化能为 $25.96 \mathrm{kcal} / \mathrm{mol}$, 过渡态有唯一虚频为 $496.6 i \mathrm{~cm}^{-1}$, 根 据过渡态的判据此过渡态是真实的(表3). 在M1中, $\mathrm{N}(1)-\mathrm{C}(2)$ 键完全断裂，二者之间的距离为 $3.048 \AA$ ，在 $\mathrm{C}(2)$ 原子处形成烯醇式结构。在TS2中，H(1)原子在 $\mathrm{O}(1)$ 和 $\mathrm{N}(3)$ 原子迁移, $\mathrm{O}(1)$ 与 $\mathrm{H}(1)$ 和 $\mathrm{H}(1)$ 与 $\mathrm{N}(3)$ 原子之 间的距离分别为 1.831 和 $1.963 \AA, \mathrm{N}(3)$ 从 $\mathrm{TiO}_{2}(101)$ 表面 $\mathrm{Ti}(5)$ 原子上脱离, $\mathrm{C}(2)$ 原子烯醇式结构上的 $\mathrm{H}(1)$ 向 $\mathrm{N}$
(3)原子转移, 形成四元环过渡态. 由于 $\mathrm{O}(1)$ 和 $N(3)$ 与 $\mathrm{TiO}_{2}(101)$ 表面 $\mathrm{Ti}(5)$ 的距离分别为 3.056 和 $2.292 \AA$, 表 明过渡态 $\mathrm{TS} 2$ 离 $\mathrm{TiO}_{2}$ 表面较远，二者之间的吸引力较 弱，导致此过程活化能高达 $96.14 \mathrm{kcal} / \mathrm{mol}$ ，该步反应 成为反应通道I的速控步骤. 从活化能上判断，迪美唑 在 $\mathrm{TiO}_{2}(101)$ 晶面上以反应通道I进行开环反应发生可 能性小.

反应通道II, 首先是羟基上的 $\mathrm{H}(1)$ 原子转移到咪唑 环的 $\mathrm{N}(3)$ 原子上, 然后 $\mathrm{C}(2)-\mathrm{N}(1)$ 键断裂, 反应通道II的 机理为 $\mathrm{Re} \rightarrow \mathrm{TS} 3 \rightarrow \mathrm{M} 2 \rightarrow \mathrm{TS} 4 \rightarrow \mathrm{P}$. 从 $\mathrm{Re} \rightarrow \mathrm{TS} 3$ 的过程中, $\mathrm{H}(1)$ 原子从 $\mathrm{O}(1)$ 原子向 $\mathrm{N}$ (3) 原子转移, 形成几乎共平 面的四元环过渡态， $\mathrm{O}(1)$ 和 $\mathrm{N}(3)$ 原子与 $\mathrm{H}(1)$ 原子之间 
表 3 迪美唑在 $\mathrm{TiO}_{2}(101)$ 晶面上反应各驻点的能量 $E$ 、相对能量 $E_{\mathrm{rel}}$ 、频率 $v$

Table 3 Energy, relative energy and frequency of each standing point in the reaction of $\mathrm{DMZ}$ on $\mathrm{TiO}_{2}(101)$ surface

\begin{tabular}{|c|c|c|c|c|c|c|c|c|}
\hline 路径 & 真空中化合物 & $E$ (a.u.) & $E_{\text {rel }}(\mathrm{Kcal} / \mathrm{mol})$ & $v\left(i \mathrm{~cm}^{-1}\right)$ & 溶液中化合物 & $E$ (a.u.) & $E_{\text {rel }}(\mathrm{Kcal} / \mathrm{mol})$ & $v\left(i \mathrm{~cm}^{-1}\right)$ \\
\hline \multirow{5}{*}{ I } & $\operatorname{Re}$ & -8106.49959 & 0 & - & $\mathrm{Re}^{\prime}$ & -8106.35096 & 0 & - \\
\hline & TS1 & -8106.45822 & 25.96 & 496.6 & TS1' & -8106.30763 & 27.19 & 565.9 \\
\hline & M1 & -8106.48577 & 8.67 & - & M1' & -8106.33494 & 10.05 & - \\
\hline & TS2 & -8106.33257 & 104.81 & 1086.1 & $\mathrm{TS}^{\prime}$ & -8106.17874 & 108.06 & 1174.7 \\
\hline & $\mathrm{P}$ & -8106.50484 & -3.29 & - & $\mathrm{P}^{\prime}$ & -8106.35082 & 0.08 & - \\
\hline \multirow{3}{*}{ II } & $\mathrm{TS} 3$ & -8106.39751 & 64.06 & 683.5 & TS3' & -8106.25717 & 58.85 & 890.5 \\
\hline & M2 & -8106.50066 & -0.67 & - & M2' & -8106.34864 & 1.45 & - \\
\hline & TS4 & -8106.47654 & 14.46 & 128.9 & TS4' & -8106.33013 & 13.07 & 128.8 \\
\hline
\end{tabular}

的距离分别为 1.148 和 $1.678 \AA$, 此步骤的活化能为 $64.06 \mathrm{kcal} / \mathrm{mol}$.

$\mathrm{M} 2 \rightarrow \mathrm{TS} 4 \rightarrow \mathrm{P}$ 过程的活化能为 $15.13 \mathrm{kcal} / \mathrm{mol}$ ，在 反应通道II中, 反应速控步骤为: $\mathrm{Re} \rightarrow \mathrm{TS} 3$, 与反应通 道I相比, 迪美唑在 $\mathrm{TiO}_{2}(101)$ 活化晶面上开环反应的关 键步骤是 $\mathrm{H}$ 的迁移过程.

迪美唑在 $\mathrm{TiO}_{2}(001)$ 表面的两条开环反应机理与 $\mathrm{TiO}_{2}$ (101)面相同，反应通道I的机理为: $\mathrm{Re}^{*} \rightarrow \mathrm{TS}^{*} \rightarrow$ $\mathrm{M}^{*} \rightarrow \mathrm{TS}^{*} \rightarrow \mathrm{P}^{*}$, 在 $\mathrm{Re}^{*}$ 中, 迪美唑分子吸附在 $\mathrm{TiO}_{2}$ 上, 咪唑环上的 $\mathrm{N}(3)$ 原子与 $\mathrm{TiO}_{2}(001)$ 表面五配位的 $\mathrm{Ti}(5)$ 原 子之间的距离为 $2.345 \AA, \mathrm{TiO}_{2}(001)$ 表面上羟基自由基 进攻迪美唑分子 $\mathrm{C}(2)$ 原子, $\mathrm{C}(2)-\mathrm{O}(1)$ 键长为 $1.397 \AA$, $\mathrm{H}(1)$ 原子与 $\mathrm{TiO}_{2}(001)$ 表面二配位的 $\mathrm{O}(2)$ 原子之间的距 离为1.934 $\AA, \mathrm{C}(2)-\mathrm{N}(1)$ 键长由 $1.370 \AA$ 增加到1.517 $\AA$, $\mathrm{N}(3)-\mathrm{C}(2)$ 键长由 $1.343 \AA$ 拉长到 $1.462 \AA$.

在过渡态 $\mathrm{TS}^{*}{ }^{*}$ 中, $\mathrm{N}(1)-\mathrm{C}(2)$ 键断裂, 键长增长至 $2.125 \AA, \mathrm{N}(3)$ 和 $\mathrm{C}(2)$ 原子与羟基上的 $\mathrm{O}(1)$ 和 $\mathrm{H}(1)$ 原子 之间的二面角为 $12.13^{\circ}$, 这 4 个原子并不共平面，表明 反应过程中, $\mathrm{C}(2)$ 原子杂化态处于开环前的 $\mathrm{sp}^{3}$ 向 $\mathrm{sp}^{2}$ 过 渡, 步骤 $\mathrm{R}^{*} \rightarrow \mathrm{TS} 1^{*}$ 的活化能为 $14.69 \mathrm{kcal} / \mathrm{mol}$, 唯一虚频 为 $180.5 i \mathrm{~cm}^{-1}$, 振动方向正确指向反应物和产物, 振动 矢量方向说明过渡态是真实的. 在 $M 1^{*}$ 中, $\mathrm{N}(1)-\mathrm{C}(2)$ 键 完全断裂, 二者之间的距离为 $2.846 \AA, \mathrm{C}(2)$ 与 $\mathrm{N}(3)$ 原子 之间恢复双键, 并在 $\mathrm{C}(2)$ 原子处形成烯醇式结构, $\mathrm{C}(2)$ $-\mathrm{O}(1)$ 键长为 $1.303 \AA$. 在 $\mathrm{TS} 2{ }^{*}$ 中, $\mathrm{H}(1)$ 原子从 $\mathrm{O}(1)$ 原子 上向 $\mathrm{N}(3)$ 原子迁移, 形成一个四元环过渡态, 此过程活 化能为 $25.15 \mathrm{kcal} / \mathrm{mol}$, 过程 $\mathrm{M1}^{*} \rightarrow \mathrm{TS} 2^{*}$ 为反应通道I的 速率控制步骤，与 $\mathrm{TiO}_{2}(101)$ 表面催化反应过程中过渡
态TS2结构不同，在过渡态TS2的分析中没有发现 $\mathrm{TiO}_{2}$ (101)晶面上 $\mathrm{O}(2)$ 原子对质子转移起到作用, 这可能是 经过渡态TS2活化能高于过渡态 $\mathrm{TS} 22^{*}$ 的一个原因.

反应通道II的机理为 $\mathrm{Re}^{*} \rightarrow \mathrm{TS}^{*} \rightarrow \mathrm{M}^{*} \rightarrow \mathrm{TS}^{*} \rightarrow \mathrm{P}^{*}$, 从 $\mathrm{Re}^{*} \rightarrow \mathrm{TS} 3^{*}$ 的过程中, $\mathrm{H}(1)$ 原子从 $\mathrm{O}(1)$ 原子上向 $\mathrm{N}(3)$ 原子迁移, 该步骤反应活化能为 $66.03 \mathrm{kcal} / \mathrm{mol}$, 反应 进行的难度大. 在 $\mathrm{M}^{*}$ 中, $\mathrm{O}(1)$ 原子与 $\mathrm{Ti}(5)$ 之间的距离 为 $2.115 \AA$, 与迪美唑分子相比, 中间体 $\mathrm{M} 2{ }^{*}$ 中 $\mathrm{C}(2)-$ $\mathrm{N}(3)$ 与 $\mathrm{C}(2)-\mathrm{N}(1)$ 的键增长, 分别为 1.534 和 $1.596 \AA$, 由 于 $\mathrm{O}(1)$ 原子失去了一个质子, $\mathrm{O}(1)-\mathrm{C}(2)$ 键长缩短为 $1.287 \AA$ ，二者之间有形成双键的趋势， $\mathrm{M}^{*} \rightarrow \mathrm{TS} 4^{*}$ 的 活化能较低, 经过渡态 $\mathrm{TS}^{*}{ }^{*}$ 的活化能为 $9.27 \mathrm{kcal} / \mathrm{mol}$, 在反应通道II中, $\mathrm{Re}^{*} \rightarrow \mathrm{TS} 3{ }^{*}$ 为速控步骤. 通过上述分 析可以看出, 迪美唑在 $\mathrm{TiO}_{2}(001)$ 表面的开环反应的最 佳反应通道为反应通道I, 即按照 $\mathrm{Re}^{*} \rightarrow \mathrm{TS} 1^{*} \rightarrow \mathrm{M} 1^{*} \rightarrow$ $\mathrm{TS} 2^{*} \rightarrow \mathrm{P}^{*}$ 进行. 我们优化所得的反应各驻点构型参数 列于表4中, 反应结构图见图7.

我们研究了 $\mathrm{TiO}_{2}$ 两个不同晶面催化迪美唑开环反 应机理, 在 $\mathrm{TiO}_{2}$ 的(101)晶面上迪美唑开环反应所需活 化能很高, 催化开环反应在热反应条件下难以进行, 在 $\mathrm{TiO}_{2}$ 的 $(001)$ 晶面上迪美唑开环反应两条通道所需 活化能比较, 反应通道I控制步骤活化能较低, 在热反 应条件下开环反应能够进行, $\mathrm{TiO}_{2}$ 的(001)晶面是活性 晶面, 在锐钛矿 $\mathrm{TiO}_{2}$ 中所占比例较低, 而(101)晶面所 占比例较大, 从我们的研究结果看出, 锐钛矿 $\mathrm{TiO}_{2}$ 的 (101)晶面结构催化迪美唑开环热反应条件下难以进 行, 若要提高锐钛矿 $\mathrm{TiO}_{2}$ 催化迪美唑开环降解的效率, 还需要增加光反应条件和光协同催化效果, 这可能就 
表 4 迪美唑在 $\mathrm{TiO}_{2}(001)$ 晶面上反应驻点构型参数 $(\AA)$

Table 4 Configuration parameters of each standing point in the reaction of $\mathrm{DMZ}$ on $\mathrm{TiO}_{2}(001)$ surface $(\AA)$

\begin{tabular}{|c|c|c|c|c|c|c|}
\hline 路径 & 化合物 & $\mathrm{N}(1)-\mathrm{C}(2)$ & $\mathrm{C}(2)-\mathrm{N}(3)$ & $\mathrm{C}(2)-\mathrm{O}(1)$ & $\mathrm{O}(1)-\mathrm{H}(1)$ & $\mathrm{N}(3)-\mathrm{H}(1)$ \\
\hline \multirow{5}{*}{ I } & $\mathrm{Re}^{*}$ & 1.517 & 1.426 & 1.397 & 1.004 & - \\
\hline & $\mathrm{TS}^{*}$ & 2.125 & 1.398 & 1.350 & 1.045 & - \\
\hline & $\mathrm{M}^{*}$ & 2.846 & 1.340 & 1.303 & 1.093 & - \\
\hline & $\mathrm{TS} 2^{*}$ & 2.986 & 1.363 & 1.260 & 2.003 & 2.035 \\
\hline & $\mathrm{P}^{*}$ & 3.227 & 1.384 & 1.249 & - & 1.028 \\
\hline \multirow{3}{*}{ II } & $\mathrm{TS}^{*}{ }^{*}$ & 1.535 & 1.474 & 1.358 & 1.484 & 1.494 \\
\hline & $\mathrm{M} 2^{*}$ & 1.596 & 1.534 & 1.287 & - & 1.024 \\
\hline & $\mathrm{TS} 4^{*}$ & 2.255 & 1.456 & 1.222 & - & 1.034 \\
\hline
\end{tabular}

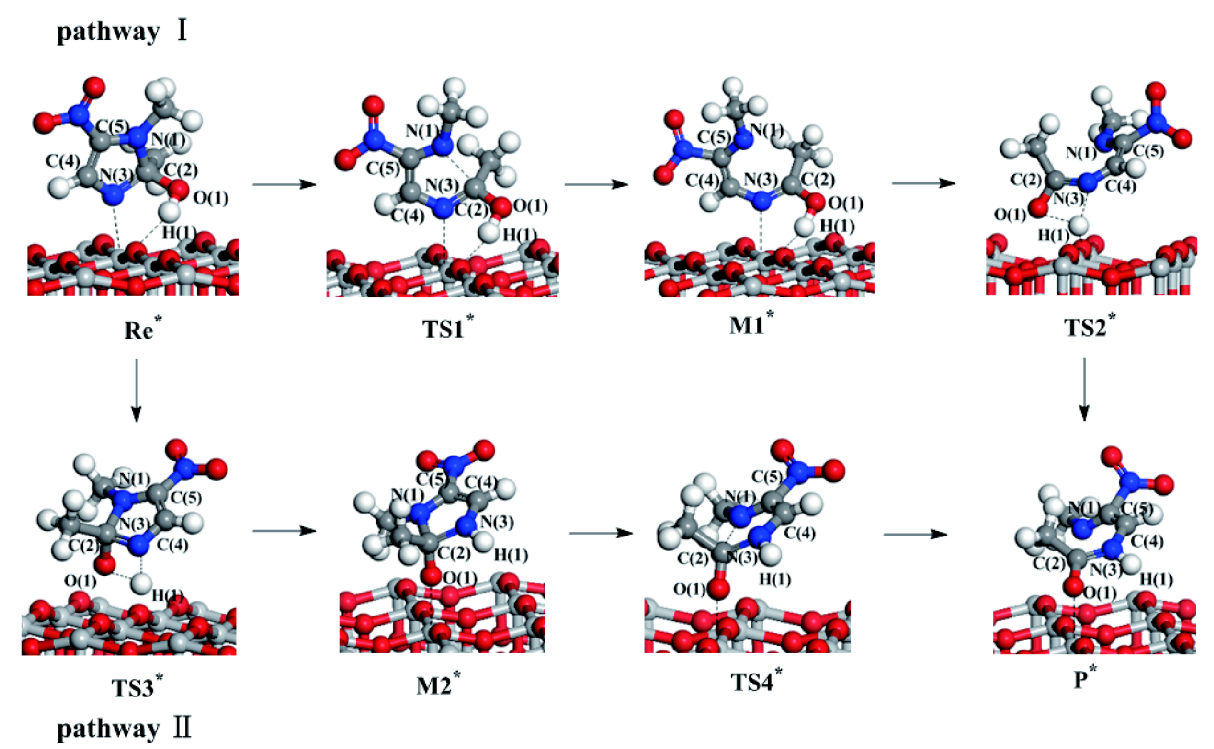

图 7 迪美唑在 $\mathrm{TiO}_{2}(001)$ 晶面上反应各驻点的构型(网络版彩图)

Figure 7 Configurations of each standing point in the reaction of $\mathrm{DMZ}$ on the $\mathrm{TiO}_{2}(101)$ surface (color online).

是目前环境处理相关催化剂的研究中，总是要同时考 察光驱动催化的原因 ${ }^{[12]}$.

我们同时研究了水溶剂模型条件下，迪美唑在 $\mathrm{TiO}_{2}(101)$ 和(001)晶面上的开环反应机理，开环反应各 驻点能量分别列于表 3 和 5 中, 迪美唑在 $\mathrm{TiO}_{2}(101)$ 和 (001) 晶面上开环反应过程中各步骤的能级图见图8. 重点分析讨论了水溶剂模型条件下迪美唑在 $\mathrm{TiO}_{2}$ (001) 晶面上的开环反应过程. 反应通道I为迪美唑在 $\mathrm{TiO}_{2}(001)$ 晶面上发生开环反应的最佳反应通道，即 $\mathrm{Re}^{* *} \rightarrow \mathrm{TS}^{* *} \rightarrow \mathrm{M1}^{* *} \rightarrow \mathrm{TS}^{* *} \rightarrow \mathrm{P}^{* *}$. 在 $\mathrm{Re}^{* *}$ 中，迪美唑分 子吸附在 $\mathrm{TiO}_{2}$ 上, 咪唑环上的 $\mathrm{N}(1)$ 原子与 $\mathrm{TiO}_{2}(001)$ 表
面五配位的 $\mathrm{Ti}(5)$ 原子之间的距离为 $2.342 \AA$, 羟基自由 基进攻 $\mathrm{C}(2)$ 原子, $\mathrm{C}(2)-\mathrm{O}(1)$ 键长为 $1.396 \AA, \mathrm{H}(1)$ 原子 与 $\mathrm{TiO}_{2}(001)$ 表面二配位的 $\mathrm{O}(2)$ 原子之间的距离为 $1.933 \AA, \mathrm{C}(2)-\mathrm{N}(1)$ 键长由原来的 $1.370 \AA$ 增加到 $1.519 \AA . \mathrm{N}(3)-\mathrm{C}(2)$ 键长由原来的 $1.343 \AA$ 拉长到 $1.464 \AA$, 所以 $\mathrm{C}(2)-\mathrm{N}(1)$ 键断裂开环的可能性最大. 在 过渡态 $\mathrm{TS} 1^{* *}$ 中, $\mathrm{N}(1)-\mathrm{C}(2)$ 键开始断裂, 键长进一步拉 长到2.191 $\AA, \mathrm{N}(3)$ 和 $\mathrm{C}(2)$ 与羟基上的 $\mathrm{O}(1)$ 和 $\mathrm{H}(1)$ 原子 之间的最大二面角为 $11.51^{\circ}$, 反应步骤 $\mathrm{Re}^{* *} \rightarrow \mathrm{TS}^{* *}$ 的 活化能为 $16.30 \mathrm{kcal} / \mathrm{mol}$, 唯一虚频为 $253.3 i \mathrm{~cm}^{-1}$, 此 时 $\mathrm{N}(1)$ 与 $\mathrm{Ti}(5) 、 \mathrm{H}(1)$ 与 $\mathrm{O}(2)$ 之间的距离分别为 2.372 和 
表 5 迪美唑在 $\mathrm{TiO}_{2}(001)$ 晶面上反应各驻点的能量 $E$ 、相对能量 $E_{\mathrm{rel}}$ 、频率 $v$

Table 5 Energy, relative energy and frequency of substances in the reaction of $\mathrm{DMZ}$ on $\mathrm{TiO}_{2}(001)$ surface

\begin{tabular}{|c|c|c|c|c|c|c|c|c|}
\hline 路径 & 真空中化合物 & $E$ (a.u.) & $E_{\text {rel }}(\mathrm{Kcal} / \mathrm{mol})$ & $v\left(i \mathrm{~cm}^{-1}\right)$ & 溶液中化合物 & $E$ (a.u.) & $E_{\text {rel }}(\mathrm{Kcal} / \mathrm{mol})$ & $v\left(i \mathrm{~cm}^{-1}\right)$ \\
\hline \multirow{5}{*}{ I } & $\operatorname{Re}^{*}$ & -8106.48166 & 0 & - & $\operatorname{Re}^{* *}$ & -8106.31235 & 0 & - \\
\hline & $\mathrm{TS}^{*}$ & -8106.45825 & 14.69 & 180.5 & $\mathrm{TS}^{* *}$ & -8106.28637 & 16.30 & 253.3 \\
\hline & $\mathrm{M}^{*}$ & -8106.47146 & 6.40 & - & $\mathrm{M}^{* *}$ & -8106.29726 & 9.47 & - \\
\hline & $\mathrm{TS}^{*}$ & -8106.43138 & 31.55 & 606.4 & $\mathrm{TS} 2^{* *}$ & -8106.27388 & 24.14 & 573.9 \\
\hline & $\mathrm{P}^{*}$ & -8106.46604 & 9.80 & - & $\mathrm{P}^{* *}$ & -8106.29255 & 12.43 & - \\
\hline \multirow{3}{*}{ II } & $\mathrm{TS}^{*}$ & -8106.37643 & 66.03 & 1626.3 & $\mathrm{TS}^{* *}$ & -8106.20456 & 67.64 & 1650.3 \\
\hline & M2* & -8106.46192 & 12.39 & - & $\mathrm{M} 2^{* *}$ & -8106.29156 & 13.04 & - \\
\hline & $\mathrm{TS}^{*}{ }^{*}$ & -8106.44714 & 21.66 & 137.3 & $\mathrm{TS}^{* *}$ & -8106.27955 & 20.58 & 256.7 \\
\hline
\end{tabular}
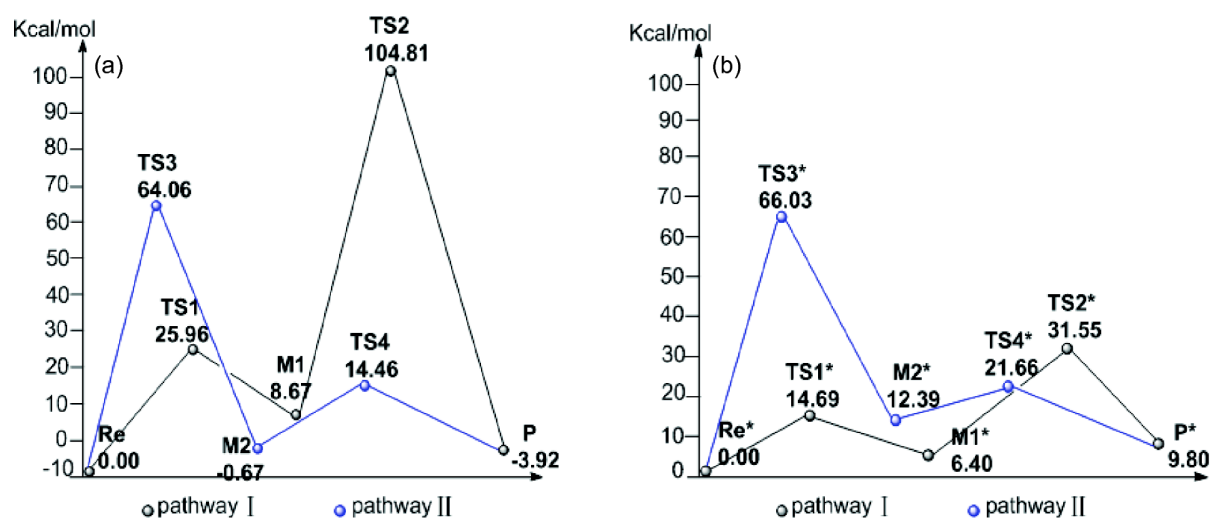

图 8 迪美㟇在反应过程中各步骤的能级图. (a) $\mathrm{TiO}_{2}(101)$ 晶面上; (b) $\mathrm{TiO}_{2}(001)$ 晶面上 (网络版彩图)

Figure 8 Energy level of $\mathrm{DMZ}$ in the reaction. (a) $\mathrm{DMZ}$ absorbed on $\mathrm{TiO}_{2}(101)$; (b) $\mathrm{DMZ}$ absorbed on $\mathrm{TiO}_{2}(001)$ (color online).

\section{$1.690 \AA ̊$.}

在 $\mathrm{M}^{* *}$ 中, $\mathrm{N}(1)-\mathrm{C}(2)$ 键完全断裂, 二者之间的距 离为 $2.818 \AA, \mathrm{C}(2)$ 与 $\mathrm{N}(3)$ 原子之间恢复双键, 并在 $\mathrm{C}(2)$ 原子处形成烯醇式结构, $\mathrm{C}(2)-\mathrm{O}(1)$ 键长为 $1.300 \AA$, $\mathrm{N}(3)$ 与 $\mathrm{Ti}(5) 、 \mathrm{H}(1)$ 与 $\mathrm{O}(2)$ 之间的距离分别为 2.416 和 $1.470 \AA$. 在 $\mathrm{TS}_{2}{ }^{* *}$ 中, $\mathrm{H}(1)$ 脱离 $\mathrm{O}(1)$ 向 $\mathrm{N}(3)$ 原子转移, 形 成一个四元环过渡态, $\mathrm{O}(1)$ 与 $\mathrm{H}(1) 、 \mathrm{H}(1)$ 与 $\mathrm{N}(3)$ 原子之 间的距离分别为 2.122 和 $2.563 \AA, \mathrm{N}(3)$ 开始从 $\mathrm{Ti}(5)$ 原子 上脱离, $\mathrm{C}(2)$ 原子烯醇式结构上的 $\mathrm{H}(1)$ 要直接向 $\mathrm{N}(3)$ 原子转移, $\mathrm{N}(3) 、 \mathrm{C}(2) 、 \mathrm{O}(1)$ 和 $\mathrm{H}(1) 4$ 个原子之间的最 大二面角为 $27.81^{\circ}$, 二面角偏大, 但是由于 $\mathrm{TiO}_{2}$ 表面 $\mathrm{O}(2)$ 原子对羟基质子的强烈吸引(二者距离为 $0.979 \AA$, $\mathrm{O}(2)$ 原子在质子转移过程中起到了类似桥梁的作用, 使反应易于进行, 此过程活化能为 $14.67 \mathrm{kcal} / \mathrm{mol}$, 有唯 一虚频 $1573.9 i \mathrm{~cm}^{-1}$, 过程 $\mathrm{Re}^{* *} \rightarrow \mathrm{TS}^{* *}$ 为反应通道I的
速率控制步骤. 水溶剂模型中, 迪美唑在锐钛矿型 (001)晶面上开环反应机理同气相相同, 但反应每步活 化能有所改变, 从反应活化能计算结果看, 在水溶剂模 型中, 降解反应每步反应活化能有所下降.

\section{4 结论}

本文采用密度泛函理论, 研究了真空和溶液条件 下，迪美唑在锐钛矿型 $\mathrm{TiO}_{2}(101)$ 和(001)晶面的吸附特 征和降解反应机理. 研究发现迪美唑均能通过氢键的 形式吸附在 $\mathrm{TiO}_{2}$ 表面. 氢键能增强吸附结构的稳定性. 最稳定吸附构型为咪唑环上的 $\mathrm{N}$ 原子吸附在 $\mathrm{TiO}_{2}$ 的 $\mathrm{Ti}(5)$ 原子上, 吸附能最大. 吸附的结果使得迪美唑的 $\mathrm{C}-\mathrm{N}$ 键长变长, 有利于后续羟基自由基的进攻发生降 解开环. 在开环降解反应机理中, 发现 $\mathrm{TiO}_{2}(101)$ 晶面 迪美唑开环降解所需反应活化能较高, 开环反应很难 
发生．而在 $\mathrm{TiO}_{2}(001)$ 表面迪美唑开环反应的活化能相 对较低，在热反应条件下可能进行．锐钛矿 $\mathrm{TiO}_{2}$ 降解 抗生素迪美唑在光辅助催化条件下有利于反应的进
行. 通过研究水溶剂化效应对反应的影响, 发现迪美唑 在锐钛矿 $\mathrm{TiO}_{2}$ 晶面降解反应活化能有所降低, 说明水 溶剂能促进降解反应的进行.

\section{参考文献}

1 Chen DM, Yu ZB, Sun L. J Environ Sci, 2015, 36: 4135-4140 (in Chinese) [陈冬梅, 喻泽斌, 孙蕾. 环境科学, 2015, 36: 4135-4140]

2 Thompson CS, Traynor IM, Fodey TL, Crooks SRH. Anal Chim Acta, 2009, 637: 259-264

3 Wang JC, Wang DJ, Yang Z, Lin L. Chin J Vet Drug, 2007, 41: 31-33 (in Chinese) [汪纪仓, 王大菊, 杨自军, 林霖. 中国兽药杂志, 2007, 41: 31-33]

4 Hu C, Deng J, Zhao Y, Xia L, Huang K, Ju S, Xiao N. Food Chem, 2014, 158: 366-373

5 Liu JL, Wong MH. Environ Int, 2013, 59: 208-224

6 Alexy R, Kümpel T, Kümmerer K. Chemosphere, 2004, 57: 505-512

7 Leung HW, Jin L, Wei S, Tsui MMP, Zhou B, Jiao L, Cheung PC, Chun YK, Murphy MB, Lam PKS. Environ Health Perspect, 2013, 121: 839846

8 Rivera-Utrilla J, Prados-Joya G, Sánchez-Polo M, Ferro-García MA, Bautista-Toledo I. J Hazard Mater, 2009, 170: 298-305

9 Yang SY. Initial processess in $\mathrm{TiO}_{2}$-assissted photodegradation of organic pollutants. Dissertation for the Doctoral Degree. Hangzhou: Zhejiang University, 2005 (in Chinese) [杨世迎. $\mathrm{TiO}_{2}$ 光催化降解有机污染物的初始步骤机理研究. 博士学位论文. 杭州: 浙江大学, 2005]

10 Van Doorslaer X, Haylamicheal ID, Dewulf J, Van Langenhove H, Janssen CR, Demeestere K. Chemosphere, 2015, 119: S75-S80

11 Gao NY, Zhang YY, Ma Y. China Environ Sci, 2013, 33: 1958-1964 (in Chinese) [高乃云, 张晏晏, 马艳. 中国环境科学, 2013, 33: 1958-1964]

12 Lamine A, Farid M, Hafida L. Int J Chem Environ Eng, 2015, 6: 150-152

13 Sánchez-Polo M, López-Peñalver J, Prados-Joya G, Ferro-García MA, Rivera-Utrilla J. Water Res, 2009, 43: 4028-4036

14 Chang R, Li CX, Meng H, Lu YZ. J Environ Eng-Asce, 2011, 5: 1950-1954 (in Chinese) [常睿, 李春喜, 孟洪, 陆颖舟. 环境工程学报, 2011, 5: 1950-1954]

15 Stepnowski P, Zaleska A. J Photochem Photobiol A-Chem, 2005, 170: 45-50

16 Hou RB, Li WW, Shen XC. Acta Phys-Chim Sin, 2008, 24: 269-274 (in Chinese) [侯若冰, 李伟伟, 沈星灿. 物理化学学报, 2008, 24: 269-274]

17 O'Rourke C, Bowler DR. J Phys Chem C, 2010, 114: 20240-20248

18 Vittadini A, Selloni A, Rotzinger FP, Grätzel M. Phys Rev Lett, 1998, 81: 2954-2957

19 Rahaman O, van Duin ACT, Goddard WA, Doren DJ. J Phys Chem B, 2011, 115: 249-261

20 Plimpton S. J Comput Phys, 1995, 117: 1-19

21 Perdew JP, Burke K, Ernzerhof M. Phys Rev Lett, 1996, 77: 3865-3868

Moellmann J, Grimme S. J Phys Chem C, 2014, 118: 7615-7621

Risthaus T, Grimme S. J Chem Theor Comput, 2013, 9: 1580-1591

Rezáč J, Riley KE, Hobza P. J Chem Theory Comput, 2011, 7: 2427-2438

Stampfl C, van de Walle CG. Phys Rev B, 1999, 59: 5521-5535

Zhu HX, Liu JM. Appl Phys A, 2014, 117: 831-839

Han X, Shao G. J Phys Chem C, 2011, 115: 8274-8282

Chen QL, Tang CQ. Acta Phys-Chim Sin, 2009, 25: 915-920 (in Chinese) [陈琦丽, 唐超群. 物理化学学报, 2009, 25: 915-920]

Dolg M, Wedig U, Stoll H, Preuss H. J Chem Phys, 1987, 86: 866-872

Bergner A, Dolg M, Küchle W, Stoll H, Preuß H. Mol Phys, 1993, 80: 1431-1441

Delley B. J Chem Phys, 1990, 92: 508-517

Halgren TA, Lipscomb WN. Chem Phys Lett, 1977, 49: 225-232

Klamt A, Schüürmann G. J Chem Soc Perkin Trans, 1993, 2: 799-805

Delley B. Mol Simul, 2006, 32: 117-123

Zhang L, Liu X, Rao W, Li J. Sci Rep, 2016, 6: 35893 


\title{
Adsorption characteristics and degradation mechanism of DMZ on photocatalyst $\mathrm{TiO}_{2}$ surface: a density functional theoretical study
}

\author{
Bohua $\mathrm{Xu}^{1}$, Fulan Zhang ${ }^{1}$, Wei Wei ${ }^{2}$, Xiao Chen ${ }^{2}$, Laicai Li ${ }^{2 *}$, Anmin Tian $^{3}$ \\ ${ }^{1}$ College of Chemistry and Chemical Engineering, Yangtze Normal University, Fuling 408100, China \\ ${ }^{2}$ College of Chemistry and Material Science, Sichuan Normal University, Chengdu 610066, China \\ ${ }^{3}$ College of Chemistry, Sichuan University, Chengdu 610064, China \\ *Corresponding author (email: lilcmail@163.com)
}

\begin{abstract}
In this article, the adsorption and degradation mechanism of dimetridazole (DMZ) on anatase $\mathrm{TiO}_{2}(101)$ and (001) crystal surfaces has been studied by density functional theory. The adsorption stability of DMZ on anatase surface was studied under vacuum and neutral water solvents. The most stable adsorption configuration was optimized by theoretical analysis. It was found that $\mathrm{DMZ}$ can be adsorbed on photocatalyst $\mathrm{TiO}_{2}$ surface under two conditions. The hydrogen bond produced in the adsorption process can enhance the stability of the adsorption structure. The stable adsorption configuration makes the $\mathrm{C}-\mathrm{N}$ bond length of DMZ longer, which is conducive to the ring opening degradation reaction. We also studied the mechanism of ring opening degradation of DMZ on two crystal planes of anatase $\mathrm{TiO}_{2}$. It was found that the reaction activation energy of the degrading reaction on $\mathrm{TiO}_{2}(101)$ crystal surface is very high, and the ring opening reaction is difficult to occur. On the (001) crystal surface, the opening of DMZ can be carried out under the condition of thermal reaction. We studied the effect of the water solvation on the degradation reaction. It was found that the activation energies of $\mathrm{DMZ}$ on the anatase $\mathrm{TiO}_{2}$ surface was reduced, indicating that the solvent conditions could promote the degradation reaction.
\end{abstract}

Keywords: density functional theory, $\mathrm{TiO}_{2}$ photocatalyst, dimetridazole, degradation, reaction mechanism

doi: $10.1360 / \mathrm{N} 032018-00127$ 